International Journal of Current Advanced Research

ISSN: O: 2319-6475, ISSN: P: 2319 - 6505, Impact Factor: SJIF: 5.995

Available Online at www.journalijcar.org

Volume 6; Issue 4; April 2017; Page No. 3447-3450

DOI: http://dx.doi.org/10.24327/ijcar.2017.3450.0290

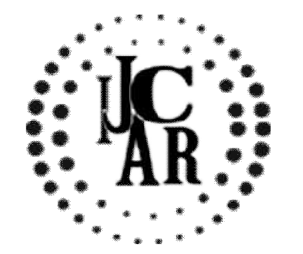

Research Article

\title{
THE ROLE OF NANOPARTICLES IN DIAGNOSIS AND MANAGEMENT OF HEALTH: A NEW APPROACH IN BIOMEDICAL SCIENCE
}

\author{
Asha Sharma* \\ Department of Applied Chemistry, Birla Institute of Technology, Mesra, Off- Campus, Deoghar, Jharkhand
}

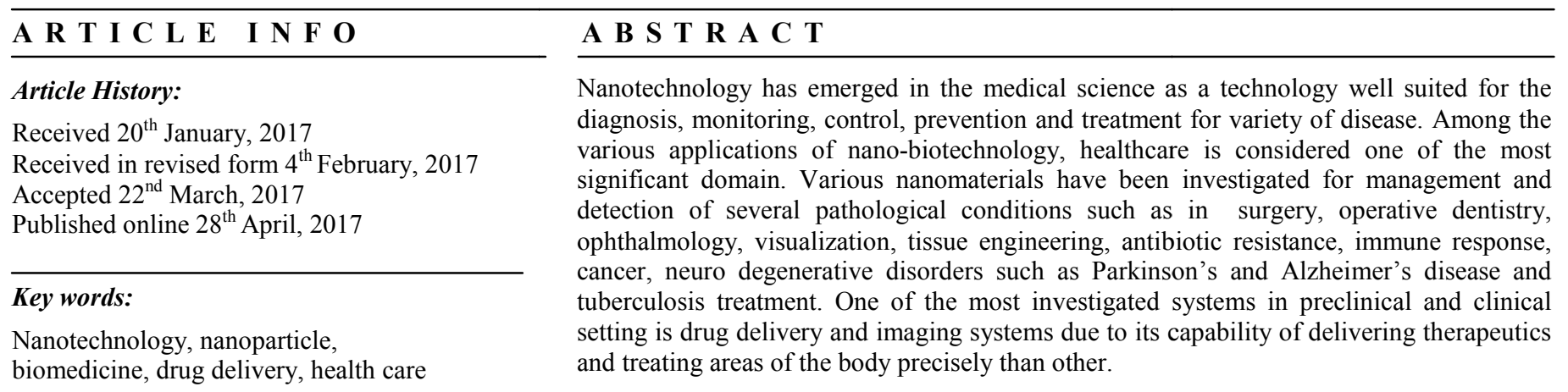

Copyright $₫ 2017$ Asha Sharma. This is an open access article distributed under the Creative Commons Attribution License, which permits

unrestricted use, distribution, and reproduction in any medium, provided the original work is properly cited.

\section{INTRODUCTION}

Nanotechnology is a multidisciplinary field that covers a huge and varied range of devices derived from engineering, physics, chemistry and biology. In a broad sense, nanotechnology can be defined as the science and engineering involved in the design, synthesis, characterization, and application of materials and devices whose smallest functional organization in at least one dimension is on the nanometer scale, ranging from a few to several hundred nanometers. A nanometer is one billionth of a meter or three orders of magnitude smaller then a micron, roughly the size scale of a molecule itself (e.g., a DNA molecule is about $2.5 \mathrm{~nm}$ long while a sodium atom is about $0.2 \mathrm{~nm}$ ). Now a day's nanomaterials have been designed for a several biomedical and biotechnological applications, including biosensors, enzyme encapsulation and neuronal nanotechnology to produce a significant scientific and technological advance in the field of biomedicine and physiology of cell. Nanomaterials are also increasingly used in several pharmaceutical industries, medical applications, environmental remediation, energy storage and efficiency, water treatment, air filtration, chemical and biological sensors, military defence, explosives, cosmetics and personal products. Various nanomaterials have been investigated for management and detection of several pathological conditions such as in surgery, operative dentistry, ophthalmology,

\section{*Corresponding author: Asha Sharma}

Department of Applied Chemistry, Birla Institute of Technology, Mesra, Off- Campus, Deoghar, Jharkhand visualization, tissue engineering, antibiotic resistance, immune response, cancer, neuro degenerative disorders such as Parkinson's and Alzheimer's disease and tuberculosis treatment. In recent years, there has been increasing evidence that nanotechnology can help to overcome many of the ocular diseases (Rai et al., 2016). Nanomaterials are also used in the area of food, to provide new tastes and flavours; functional foods; hygienic food processing and packaging, extended shelf-life of products and reduced agrochemicals, colours, flavours and preservatives (Chaudhry, 2012).

\section{Imaging and diagnosis}

Molecular imaging procedures involve mostly an imaging device and an imaging agent (probe). These probes necessitate two key characteristics: a property that facilitates their accumulation at the site of interest and a property that allows them to be imaged. Nanoparticulate probes have demonstrated significant advantages over single molecule-based contrast agents. Molecular imaging is an important discipline to detect, quantify, and display in vivo and in vitro changes at molecular and cellular level in biomedical science. Several nanoparticles have been used for diagnostics, most commonly gold nanoparticles, quantum dots (QDs) and magnetic nanoparticles Small sections of DNA can be attached to gold particles smaller than $13 \mathrm{~nm}$ in diameter. These attach onto a sensor surface only in the presence of a complementary target. Quantum dots are inorganic fluorophores offering significant advantages over traditionally used fluorescent markers. They have high sensitivity (brighter imaging signals), broad excitation spectra, stable fluorescence with simple excitation and do not require lasers. Their infrared colours enable whole 
blood assays and have a wide range of applications for molecular diagnostics and genotyping (Najar, 2012). The NP based dye are more efficient comparatively to conventional fluorophores (Bruchez et al. 1998). In a study Wu et al. (2003) found that quantum dot-based immunofluorescent labeling of the cancer marker Her2 was more efficient than conventional fluorophores in labeling different target cell surface receptors, cytoskeleton, nuclear antigens, and other intracellular organelles. Magnetic nanoparticles are used as labelling molecules for bioscreening. Superparamagnetism is a form of magnetism that appears in ferromagnetic or ferrimagnetic (types of permanent magnetism) nanoparticles. Superparamagnetic nanoparticles are useful for cell-tracking cells and for calcium sensing. A family of calcium indicators for MRI is formed by combining a powerful superparamagnetic iron oxide (SPIO) nanoparticle-based contrast medium with the versatile calcium-sensing protein calmodulin and its target (Rajasundari and Ilamurugu 2010). Superparameganetic nanoparticles measuring 2-3nm have been used in conjunction with MRI to reveal small and otherwise undetectable lymph node metastases.

\section{Drug delivery}

To overcome the problems of gene and drug delivery, nanotechnology has gained interest in recent years. Nanosystems with different compositions and biological properties have been extensively investigated for drug and gene delivery applications. Nanoparticles used as drug delivery vehicles are generally $<100 \mathrm{~nm}$ in at least one dimension, and consist of different biodegradable materials such as natural or synthetic polymers, lipids, or metals. Nanoparticles are taken up by cells more efficiently than larger micromolecules and therefore, could be used as effective transport and delivery systems. In drug delivery, several approaches are currently being tested for better sitespecific delivery of an effective dose using liposomes, polymeric micelles, dendrimeres, ceramic NPs, iron oxide, proteins, covalent binding, adsorption, conjugation, and encapsulation methods (Moghimi et al. 2005).

There are several techniques used for the drug delivery. The choice of system depends on the way they bind with the drug and the type of drug treatment. Nanocapsules, Liposomes, Buckyball (spherical nanoparticles can carry more than one drug at a time), Dendrimers (tree-shaped synthetic nanomolecules that carry drugs in the tips of the branches). They are useful in the treatment of diseases such as cancer and other diseases where monotherapy can lead to drug resistance.

\section{Nanotechnology and disinfectants}

The antibacterial properties of metals such as silver, zinc and copper have been known for centuries and this has been exploited in modern medicine for infection control (Weber and Rutala 2001). Engineered nanomaterials display significant advantages due to their unique nanostructure, along with their tuneable properties for the designed application. Silver nanoparticles (Ag-NPs) have drawn attention due to their use as potent bactericidal. Silver is known for combating pathogenic microorganism. The increasing resistance of bacteria to antibiotics has resulted in renewed interest in silver as a disinfectant. Silver ions are used as antiseptic (Lansdown et al., 2002). Silver ion blocks the enzyme active site and inhibit oxygen metabolism in cell membrane and block cell division. Bacteria are not expected to develop resistance to silver, owing to the diversity of the working mechanisms. Silver nanoparticle are used as a disinfectant due to its large exposed sites to the environment (Shrivastava et al., 2007). Titanium dioxide is used as bactericidal its activity is based on a photocatalytic effect. Under the influence of ultraviolet radiation and in the presence of water and oxygen, the particles form extremely reactive molecules (radicals), such as hydroxyl and perhydroxyl radicals, which kill microorganisms (Maness et al., 1999).

\section{Nanotechnology and Reproductive health}

The role of nanotechnology in reproductive medicine is a continuously developing field, solely aims to endow the patients with the best chances to safely conceive and deliver a healthy offspring at a chosen time in their lives. Now a day's infertility has become ominous problem. On an average, about $10 \%$ of all couples face difficulty in starting a family and this creates a feeling of great personal failure. According to WHO (1999) 60-80 million couples are infertile worldwide. Infertility affects $15 \%$ of the married couples and in about $50 \%$ of cases male factor is the predominant causative factor (Seshagiri, 2001; Sharlip et al., 2002). There are several causes which affects fertility potential i.e., increased body weight (BMI), age at parenthood, occupational exposure and life style. Increased BMI is also associated with decreased sperm functions and serum FSH and testosterone level (Asha et al., 2015). Paternal exposures to toxicants before conception could also contributes to the genetic defects in subsequent generations, expressed as congenital abnormalities in the offspring (Ansari et al., 2007). The age is intimately related to decreasing in sperm motility and vitality, whereas, least effect is observed on sperm count. In a study we observed that the occupationally, the highest prevalence of abnormal semen quality was noticed in farmers. Lifestyle of alcohol consumption and smoking further diminishes the semen quality (Asha, 2014). The genetic basis of infertility has received increasing recognition in recent years. It is now obvious that genetic etiology for infertility is an important cause of disrupted spermatogenesis. Sperm DNA is known to contribute one half of the genomic material to offspring. The field of reproductive medicine has been increasingly fuelled by the progress in fundamental reproductive biology, investigating the structure and function of reproductive tissues and fine mechanisms of gametogenesis, fertilisation, implantation and early embryo development (Gianaroliet al., 2012). With respect to STDs or STIs, RISUG has also shown primary evidence to work against HIV, and RISUG-coated IUDs do not allow microbes in its vicinity when placed inside the body after child birth (Guha, 2005). Although nanotechnology does not appear to play a direct role in infertility treatment, indirectly it can play an immense role by helping in early, low-cost and accurate detection of disease sites with smart sensors, detection of hormone levels and noninvasive imaging of nanomedicines placed in the reproductive tube. In today's world, many reproductive diseases go undiagnosed or misdiagnosed, leading to even more complications. Nanotechnology may improve the sensitivity, selectivity, speed, cost, and convenience of diagnosis (Yager 
et al., 2006). Nanomarkers give optical contrast and molecular specificity to optical imaging of reproductive organelles and help detect cell organelles as well as suborganelles. Solution biomarkers are an important form of nanotechnology that is able to attach itself to various diseased cells inside the reproductive system enabling to analyze it and treat the person accordingly (Horne et al., 2010).

\section{Adverse effect of nanomaterials}

Nanotechnology will have a reflective impact on several of aspects of human life including environmental decontamination, water purification, cheaper electricity, and better disease treatment modalities. Humans have always been exposed to tiny particles via dust storms, volcanic ash, and other natural processes, and that our bodily systems are well adapted to protect us from these potentially harmful intruders. In addition to being able to cross cell membranes, reach the blood and various organs because of their very small size, nanoparticles of any material have a much greater surface to volume ratio (i.e. the surface area compared to the volume) than larger particles of that same material. Therefore, relatively more molecules of the chemical are present on the surface. This may be one of the reasons why nanoparticles are generally more toxic than larger particles of the same composition. Adverse effects of nanoparticles on human health depend on individual factors such as genetics and existing disease, as well as exposure, and nanoparticle chemistry, size, shape, agglomeration state, and electromagnetic properties. Inhaled particulate matter can be deposited throughout the human respiratory tract, and an important fraction of inhaled nanoparticles deposit in the lungs. Nanoparticles can potentially move from the lungs to other organs such as the brain, the liver, the spleen and possibly the foetus in pregnant women. Data on these pathways is extremely limited but the actual number of particles that move from one organ to another can be considerable, depending on exposure time. Even within the nanoscale, size is important and small nanoparticles have been shown to be more able to reach secondary organs than larger one Animal and human studies show that inhaled nanoparticles are less efficiently removed than larger particles by the macrophage clearance mechanisms in the lungs, causing lung damage, and that nanoparticles can translocate through the circulatory, lymphatic, and nervous systems to many tissues and organs, including the brain [Buzea $\mathrm{C}$, et al 2007]. Nanoparticles may be used effectively to deliver genes to cells, to treat cancer, as well as in vaccination. The use of nanoparticles as drug carriers may reduce the toxicity of the incorporated drug but it is sometimes difficult to distinguish the toxicity of the drug from that of the nanoparticle.

\section{Summary}

Nanotechnology is an exciting novel multi-disciplinary field, hastily expanding across a variety of industries, including biotechnology, pharmaceutical synthesis and also in clinical medicine. There is increasing anticipation that nanotechnology can dramatically alter routine approaches to the detection and treatment of a variety of diseases, although the revolutionary and controversial nature of this discipline remains a matter of discussion. In the future, we could imagine a world where medical nanodevices are routinely implanted or even injected into the bloodstream to monitor health. Nanotecnology touches on numerous established disciplines, about elements of physiology, biotechnology, chemistry, physics, electrical engineering, and materials science, this broad sweep of knowledge is difficult for any one investigator to master fully. Therfore nanotechnology in medicine faces enormous technical hurdles in that long delays and numerous failures are inevitable. This is because the effort needed to produce nanoscale biomedical or therapeutic devices is highly interdisciplinary. The breadth of the effort constitutes just one of the major barriers to entry in the field. Other challenges include inadequate funding, the raw complexity of biology, the fashion in which biologists hold and distribute information, and cultural differences between engineers and biological scientists. Likewise, it should not be taken for granted the dangers and negative consequences of nanobiotechnology when applied in warfare, application in energy generation when and wherever it strikes, or the risks associated with nanoparticles in blood circulation. Success requires more investment induced by cost-effectiveness analyses and business plans based on clinical data, public education based on nanotoxicology studies, smart regulatory reform in the areas of testing, market entry and liability.

\section{Reference}

1. Rai M, Ingle AP, Gaikwad S, Padovani FH, Alves M (2016). The role of nanotechnology in control of human diseases: perspectives in ocular surface diseases. Crit Rev Biotechnol: 36(5):777-87

2. Chaudhry Q (2012). Current and projected applications of nanomaterials. WHO Workshop on Nanotechnologyand Human Health: Scientific Evidence and Risk Governance. Bonn, Germany, 1011.

3. Nazar H (2012). The use of nanotechnology in disease diagnosis and molecular imaging Pharmaceutical Journal; 290:115.

4. Bruchez M Jr, Moronne M, Gin P, Weiss S, Alivisatos AP. (1998). Semiconductor nanocrystals as fluorescent biological labels. Science 281(5385):2013-2016.

5. Wu X, Liu H, Liu J, Haley KN, Treadway JA, Larson JP, et al. (2003). Immunofluorescent labeling of cancer marker Her2 and other cellular targets with semiconductor quantum dots. Nat Biotechnol 21(1):4146.

6. Rajasundari K, Ilamurugu K (2010). Nanotechnology and its applications in medical diagnosis. Journal of Basic Chemistry and Applied Chemistry; 1:26-32.

7. Moghimi SM, Hunter AC, Murray JC. (2005). Nanomedicine: current status and future prospects. FASEB J 19(3):311-330.

8. Weber DJ, Rutala WA (2001). Use of metals as microbicides in preventing infections in healthcare. In: Block SS, editor. Disinfection, sterilization, and preservation. Philadelphia: Lippincott Williams and Wilkins; 415-427.

9. AB. Lansdown, (2002). "Silver. I: its antibacterial properties and mechanism of action," Journal of Wound Care; 11 (4): 125-130.

10. S. Shrivastava, T. Bera, A. Roy, G. Singh, P. Ramachandrarao, and D. Dash (2007). "Characterization of enhanced antibacterial effects of novel silver nanoparticles," Nanotechnology, $18(22) 225103-22511$ 
11. PC. Maness, S. Smolinski, D. M. Blake, Z. Huang, E. J. Wolfrum, and W. A. Jacoby (1999). "Bactericidal activity of photocatalytic $\mathrm{TiO} 2$ reaction: toward an understanding of its killing mechanism," Applied and Environmental Microbiology; 65(9): 4094-4098.

12. WHO (1999). Laboratory Manual for the Examination of Human Semen and Sperm-Cervical Mucus Interaction. 4th edition, Geneva, World Health Organization 1999.

13. Seshagiri PB (2001). Molecular insights into the causes of male infertility. J Biosci 26:429-435.

14. Sharlip ID, Jarow JP, Belker AM, Lipshultz LI, Sigman M, Thomas AJ, Schlegel PN, Howards SS, Nehra A, Damewood MD, Overstreet JW and Sadovsky R (2002). Best practice policies for male infertility. Fertil Steril 77:873-882.

15. Asha Sharma, Ansari, A.S., and Lohiya, N.K. (2015). Impact of body mass index (BMI) on sperm functional parameters and serum hormonal profile of infertile males. International Journal of Health Sciences and Research. 5(2): 315-323.

16. Ansari, A.S., Sharma, A. and Lohiya, N.K. (2007). Does paternal occupation and life style affects embryo quality? Journal of Endocrinology and Reproduction 11(1):15-22.
17. Asha Sharma (2014). Impact of age, verified occupation and lifestyle on semen parameters of infertile males in Jaipur: A preliminary study. International Journal of Health and Allied Sciences 3(4):278- 283.

18. Gianaroli L, Racowsky C, Geraedts J, Cedars M, Makrigiannakis A, Lobo RA (2012). Practices of ASRM and ESHRE: a journey through reproductive medicine. Fertil Steril; 98:1380-94.

19. Guha SK (2005). RISUG ${ }^{\text {TM }}$ (reversible inhibition of sperm under guidance)-an antimicrobial as male vas deferens implant for HIV free semen. Med Hypotheses; 65:61-4

20. Yager P, Edwards T, Fu E, Helton K, Nelson K, Tam MR, et al. (2006). Microfluidic diagnostic technologies for global public health. Nature; 442:412-18.

21. Horne AW, Duncan WC, Critchley HO (2010). The need for serum biomarker development for diagnosing and excluding tubal ectopic pregnancy. Acta Obstet Gynecol Scand;89:299-301

22. Buzea C, Pacheco, Robbie K. (2007). Nanomaterials and nanoparticles: sources and toxicity. Biointerphases.;2(4): 17-71

\section{How to cite this article:}

Asha Sharma (2017) ' The Role Of Nanoparticles In Diagnosis And Management Of Health: A New Approach In Biomedical Science', International Journal of Current Advanced Research, 06(04), pp. 3447-3450.

DOI: http://dx.doi.org/10.24327/ijcar.2017.3450.0290 